\title{
An Investigation on the Effects of Different Stratifications on Negatively Buoyant Jets
}

\author{
Simone Ferrari ${ }^{1, *}$, Maria Grazia Badas $^{1}$, and Giorgio Querzoli ${ }^{1}$ \\ ${ }^{1}$ DICAAR - Dip. Ingegneria Civile, Ambientale e Architettura, University of Cagliari, via Marengo 2, 09123 Cagliari, Italy
}

\begin{abstract}
Negatively buoyant jets develop when fluids are released upwards into a lighter fluid or, vice versa, downwards into a heavier fluid. There are many engineering applications, such as the discharge, via submerged outfalls, of brine from desalination plants into the sea. Some concerns are raised about the potential negative environmental impacts of this discharge. The increase in salinity is the major cause for environmental impact, as it is very harmful to many marine species. The diffusers for brine discharge are typically inclined upwards, to increase the path before the brine reaches the sea bottom, as it tends to fall downwards driven by negative buoyancy. The negatively buoyant jet that develops conserves axisymmetry only when released vertically, so that it is not possible to use the well-known equations for axisymmetric jets. The main target of this paper is to investigate on a laboratory model the effects of different stratifications on the features of negatively buoyant jets. This has been done via a LIF (Light Induced Fluorescence) technique, testing various release angles on the horizontal and densimetric Froude numbers. Except for the initial stage, a different widening rate for the upper boundary and the lower boundary has been highlighted.
\end{abstract}

\section{Introduction}

Negatively buoyant jets (NBJs) develop when fluids are released upwards into a lighter fluid or, vice versa, downwards into a heavier fluid.

NBJs are implied in many fields, from the passive containment cooling system of nuclear power plants (Wang et al., 2017 [1]) to the volcanic eruption columns (Kaminski et al. 2005 [2]), see Ferrari and Querzoli, 2010 [3], for a list. In particular, the discharge of brine from desalination plants is one of the most investigated (see, for instance, Cipollina et al., 2004 [4]; Kikkert et al., 2007 [5], Besalduch et al., 2013 [6], Ferrari and Querzoli, 2015 [7], Crowe et al., 2016 [8], Nikiforakis et al., 2017 [9]), because of the concerns linked to the potential negative environmental impacts of the brine discharge (Campbell et al., 2005 [10]): this is mainly due to the increase in salinity which brine discharges impose on the receiving body, harmful to a lot of marine species, in particular to the Posidonia Oceanica (see Latorre, 2005 [11]; Sànchez-Lizaso et al., 2008 [12]).

The typical configuration for marine brine discharge is a submerged outfall equipped with upward-inclined diffusers: in this way, the path followed by the brine is increased, as brine is driven downwards by negative buoyancy and the path is delimited by the sea bottom (e.g. Roberts and Toms, 1988 [13], and Jirka, 2008 [14]). Even if, as above showed, there have been several investigations about NBJs, the high complexity of the physical phenomenon is not yet fully understood. As a matter of fact, NBJs conserve axisymmetry only as long as they are vertically released, so it is not possible to use the well-known equations for axisymmetric jets. As this lack in axisymmetry is mainly due to the different conditions of stratifications in the upper and lower boundaries of NBJs, the main target of this paper is to investigate the different effects of these stratifications on the features of NBJs.

\section{Dimensional Analysis}

When a NBJ is released from an upward-inclined diffuser, the flow is driven from two sources: one of momentum and one of negative buoyancy. As a consequence (see Figure 1), if the initial part is driven mostly by the momentum (more "simple-jet-like"), as far as the fluid moves away from the outlet, the buoyancy prevails and the axis bends down (more "plume-like"). As stated by List, 1979 [15], when the receiving fluid is at rest, the only parameters characterizing the boundary conditions are the initial flux of volume $Q$, the initial flux of momentum $M$ and the initial flux of buoyancy per unit mass $B$ : performing a dimensional analysis, two length scales $l_{Q}$ and $l_{M}$ can be obtained. The former, i.e. $l_{Q}$, gives the magnitude of the distance from the orifice where the jet becomes independent from the initial conditions:

$$
l_{Q}=\frac{Q}{\sqrt{M}}
$$

\footnotetext{
Corresponding author: ferraris@unica.it
} 


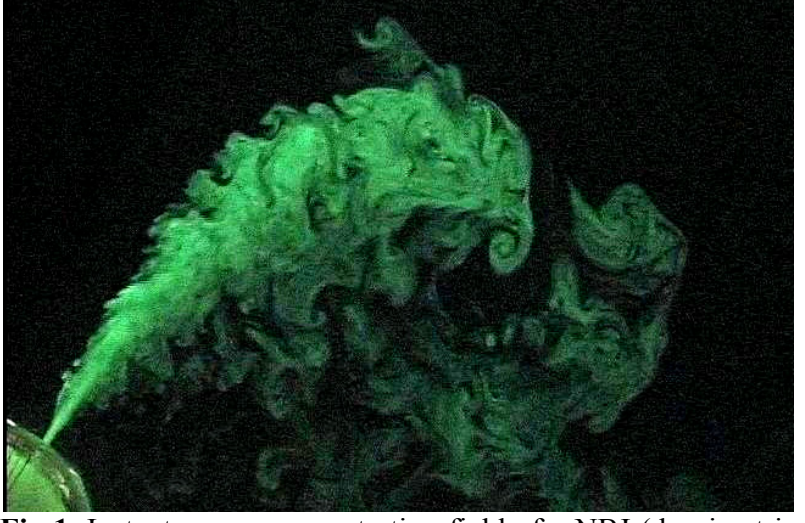

Fig 1. Instantaneous concentration field of a NBJ (densimetric Froude number Fr: 14.8; release angle to the horizontal $\theta: 55^{\circ}$ ).

In the case of a circular outlet, such as the one employed in the experiments presented here, $l_{Q}$ becomes:

$$
l_{Q}=\sqrt{\pi / 4} D \cong D
$$

The latter, i.e. $l_{M}$, gives the magnitude of the distance from the orifice where the plume-like behaviour prevails:

$$
l_{M}=\frac{M^{3 / 4}}{\sqrt{B}}
$$

From the ratio of $l_{Q}$ to $l_{M}$, a non-dimensional number, the Richardson number $R i$, measuring the relative importance of buoyancy and momentum, can be obtained:

$$
R i=\frac{l_{Q}}{l_{M}}
$$

The most important non-dimensional parameter for NBJ is usually considered the densimetric Froude number $F r$, i.e. the ratio of inertia to buoyancy:

$$
F r=\frac{U}{\sqrt{g \frac{\rho_{D I S C}-\rho_{R E C}}{\rho_{\mathrm{REC}}} D}}
$$

where $U$ is the mean initial jet velocity, $\rho_{D I S C}$ the discharged fluid density, $\rho_{R E C}$ the receiving fluid density and $D$ the outlet diameter. $F r$ an $R i$ are inversely proportional:

$$
F r=\sqrt[4]{\frac{\pi}{4}} R i^{-1}
$$

In addition to $F r$, the non-dimensional parameters influencing NBJs are the Reynolds number

$$
\operatorname{Re}=\frac{U D}{v}
$$

where $v$ is the kinematic viscosity of the brine, and the angle to the horizontal $\theta$. As $\theta$ measures the misalignment between $M$ and $B$, a NBJ will be axisymmetric only if $\theta$ is $\pm 90^{\circ}$.

\section{Experimental set-up and data elaboration}

Experiments were carried out in the Hydraulics Laboratory of the DICAAR, University of Cagliari. In particular, a $30 \mathrm{~cm}$ wide, $21 \mathrm{~m}$ long flume was employed. The flume was filled with tap water to simulate the sea at rest and a solution of water, sodiumsulphate (a low corrosive salt employed to increase the density of the solution) and fluoresceine (a fluorescent salt) was used to simulate the brine. The solution flew (through a pipe) from a constant head tank (supplied by a closed hydraulic circuit) to a cylindrical vessel, simulating a portion of a pipe laid down the sea bottom, with a sharp-edged orifice on its lateral wall. This was done to simulate a widely employed configuration for the marine outfall design (see, for instance, Wright et al., 1982 [16]; Avanzini, 2006 [17]).

To allow measurements via a Digital Image Analysis (DIA) technique (see below), the NBJ middle vertical section was lighted by a $4 \mathrm{~mm}$ thick light sheet and a video-camera, orthogonal to the light sheet, filmed the experiments at a frequency of $25 \mathrm{~Hz}$ and a resolution of $720 \times 576$ pixels. The flow was investigated by means of LIF (Light Induced Fluorescence), a DIA technique: in Fig. 1, the reader can see an example of an instantaneous image, where a light NBJ is above a dark background. With the employed concentrations, the concentration field is proportional to the light intensity, so a quantitative measure of the concentration is allowed. Digital Image Analysis (DIA) techniques have many advantages, if compared to the traditional techniques employing physical point probes: DIA are non-intrusive and quasi-continuous in space, as every pixel on the camera sensor works as a single probe (see Ferrari, 2017 [18] for a review). The LIF codes were designed inhouse by the Hydraulics Group of the University of Cagliari, which has a considerable expertise in the development of DIA techniques to measure concentrations (Ferrari and Querzoli, 2010 [3], Ferrari et al., 2016 [19]), velocity (Besalduch et al., 2013 [6] and 2014 [20], Ferrari et al, 2017 [21]) and accelerations (Ferrari et al, 2007 [22], Ferrari and Rossi, 2008 [23], Ferrari et al, 2008 [24], Lardeau et al., 2008 [25], Rossi et al, 2009 [26]) in the environmental (such as in the present paper), biomedical (Badas et al., 2015 [27], Espa et al, 2012 [28]) or civil and industrial fields (Badas et al., 2017 [29]).

Experiments we were performed with $\theta=45^{\circ} \div 90^{\circ}$ and $F r=4.9 \div 40.2$ : by this way, the whole field of practical interest was investigated. Moreover, $Q$ was 
kept constant and high enough to have a $R e$ larger than its critical value for the apparatus.

Under the hypothesis of ergodicity, the statistics of mean and variance of the concentration field were obtained by time averaging. On Fig. 2 and 3, the mean and the variance of the concentration field are shown. The mean concentration is normalized by the initial concentration, the variance by the squared maximum concentration. The jet axis was assumed to be coincident with the locus of the concentration maxima on jet crosssections. Detailed information on these parameters, as well as on the experimental set-up and data elaboration, can be found on Ferrari and Querzoli, 2010 [3] and on Ferrari et al., 2016 [19].

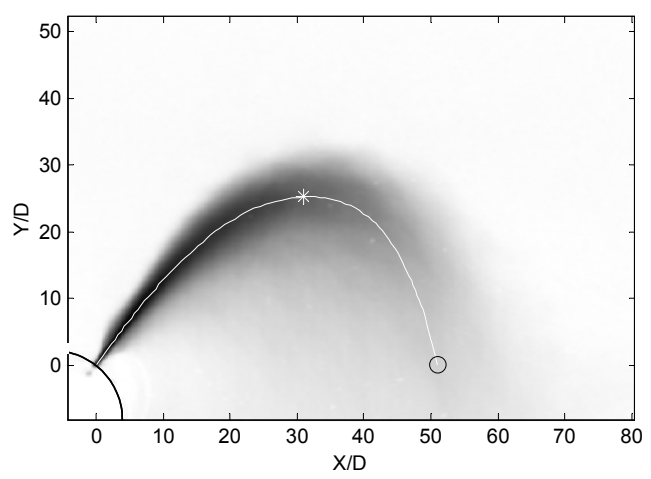

Fig. 2. Map of the non-dimensional mean concentration for the same NBJ of Fig. 1; the white line is the jet axis, the white asterisk is the point of maximum height and the black circle is the impact point.

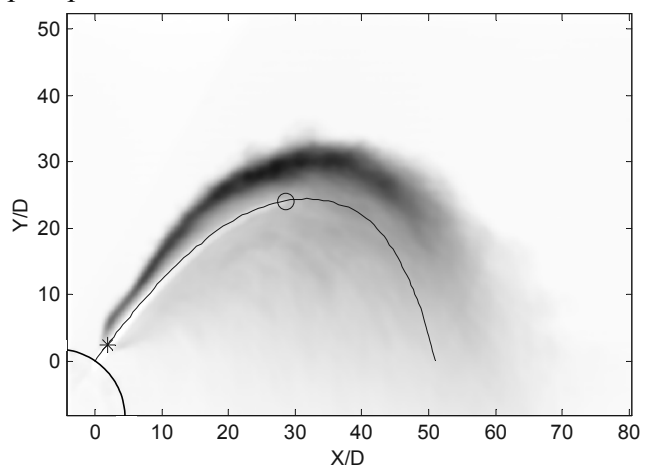

Fig. 3. Map of the variance of the concentration, nondimensionalised by the square of the maximum concentration, for the same NBJ of Fig. 1; the black line is the jet axis, the black asterisk is the point of onset of the Kelvin-Helmholtz instability and the black circle is the point of transition to turbulence of the whole NBJ.

\section{Results}

This paragraph is organised as follows: first of all, a discussion about the different stratifications, stable on the upper region and unstable in the lower region of the NBJ, will be given; then, the concentration profiles, orthogonal to the NBJ axis, at various distances from the outlet will be shown, in order to discuss their shape, with particular regard to the Gaussian shape hypothesis often employed in the study of these phenomena; lastly, the focus will be on the widening features of the NBJ upper and lower region.

\subsection{The different stratifications on the NBJ boundaries}

From Figures 1, 2 and 3, we can see that NBJs released upwards are characterised by an initial region with a width similar to the outlet diameter $D$ : afterwards, the onset of Kelvin-Helmholtz $(\mathrm{KH})$ instabilities (in the region pointed out by a black asterisk on Figure 3) causes a sudden widening, as the consequent billows drag external fluid into the NBJ, resulting in mixing and dilution. This dilution, as demonstrated by the reduction in the grey intensity along the axis on Figure 2, grows along the trajectory followed by the jet.

On Figure 2, we can see that the mean concentration field of a NBJ is symmetrical in the first part of the jet, where the flow is driven essentially by $M$; then, as $B$ becomes relevant, the span-wise sections become neither symmetrical nor self-similar (see chapter 4.2). As a consequence, above the axis there is a narrow region characterized by high upwards concentration gradients, whilst below the axis there is a wide region of smooth variations. This is due to the fact that, on the upper boundary, the local stable stratification ( $M$ and $B$ have different directions) allows a full development of compact KH billows, followed by their breaking; on the contrary, the local unstable stratification on the lower boundary ( $M$ and $B$ have the same direction) tends to transform the growing waves into downward plumes.

On Figure 3, the variance field shows the lowest values in the core region of the NBJ, close to the axis, values that increase towards the upper and lower boundaries. The stable stratification at the upper boundary of the NBJ has the consequence that the maximum variance values are located at the upper, external region, where the $\mathrm{KH}$ waves develop upwards but rapidly break, because of the downwards $B$ contrasting their growth. On the opposite, the unstable stratification at the lower boundary of the NBJ has the consequence that the downwards-developing, plume-like structures are driven away from the NBJ core by the buoyancy, with more distributed but less intense concentration fluctuations.

\subsection{Shape and Gaussianity of the concentration cross-sectional profiles}

As previously stated, when the receiving fluid is at rest, a NBJ conserves axisymmetry only if it is released vertically ( $M$ and $B$ have the same direction) but, if the discharge is done with a different $\theta$, the NBJ rapidly loses its symmetry. This is clearly visible in Figure 4 and 5 , where cross-sectional profiles of $C / C_{0}$ (the nondimensional mean concentration $C$, normalized by the initial mean concentration $C_{0}$ ), are shown; $R / D$ is the non-dimensional coordinate orthogonal to the jet axis (positive values of $R / D$ identify the lower portion of the jet, while negative ones identify the upper portion). The legend shows the non-dimensional distance $S / D$, measured from the outlet along the axis, where the profiles are measured. The different shape between the upper and lower region increases with increasing $S / D$. 
The measured points on the concentration profiles (circles, asterisks, crosses and triangles) are shown together with the best-fit Gaussian curves (in a leastsquares sense), are shown on Figure 6; the NBJ upper region is on the left-hand side (negative $R / D$ ), the lower one on the right-hand side (positive $R / D$ ).

This graph highlights another difference between the upper and lower regions: the concentration profiles have a Gaussian shape, both in the lower and upper part, in the initial region of the NBJ, close to the outlet (solid lines in Figure 6), even if they are not identical. As $S / D$ grows, the upper region conserves a Gaussian shape (dashed and dotted lines on the left), whilst the lower region loses its Gaussian shape (as shown by the dashed and dotted lines on the right that do not correctly fit the experimental data). When the NBJ becomes to an end (last section, $S / D=50$ ), both the profiles have a Gaussian shape (dash-dot lines), but again with different coefficients.

We can consequently state that an inclined NBJ cannot be considered as a Gaussian phenomenon.

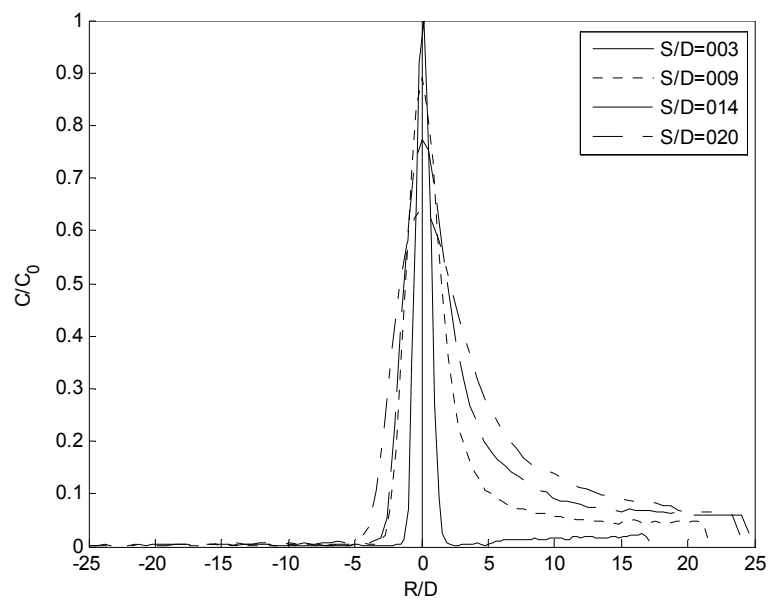

Fig. 4. cross-sectional non-dimensional concentration profiles for a NBJ with $\theta=45^{\circ}, F r=8.0$; positive values of $R / D$ identify the lower portion of the NBJ, negative ones identify the upper portion; $S / D$ is the non-dimensional distance from the outlet measured along the axis; $S / D=3 \div 20$.

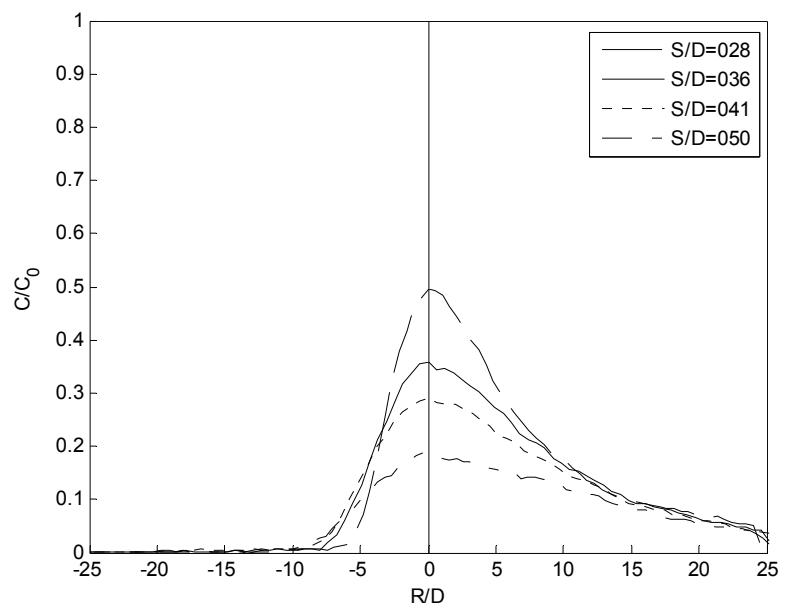

Fig. 5. cross-sectional non-dimensional concentration profiles for the same NBJ of Fig. $4 ; S / D=28 \div 50$.

\subsection{NBJ widening features}

To study the widening features of inclined NBJs, Figures 7, 8, 9 and 10 show the experimental data for the widening of the upper boundary (red asterisks) and lower boundary (blue circles) of NBJs, taking into account all the $\theta$ in the range $45^{\circ} \div 70^{\circ}$ and $F r$ of 30.8 (Fig.7), 23.8 (8), 14.8 (9) and $8.0(10)$. The data for the highest $\theta$ have been not taken into account because at those $\theta s$ NBJs experiences the so called re-entrainment. Re-entrainment is the mixing of the NBJ fluid with itself instead of with external fluid; under these conditions, there is not a sharp separation between the uprising and descending branches of the NBJs, as shown by Ferrari and Querzoli, 2010 [3], and consequently it is not possible to define the lower boundaries of the NBJs. The widening is computed as the $R / D$ where the concentration decreases to a fraction $1 / \mathrm{e}$ of the maximum concentration on that particular profile.

The NBJs with the highest $F r$ (30.8 on Figure 7) experience the largest values of $S / D$, because they are the lightest and, as a consequence, they cover the longest distance before falling down to the sea bottom; of course, exactly the opposite happens for the heaviest NBJs $(F r=8.0$ on Figure 10$)$, that cover the shortest path. For $S / D$ lower than the length scale $l_{M}$ (in the range between 29, for $F r=30.8$ and 7.6, for $F r=8.0$ ), the widening is similar for the upper and lower regions, as the NBJ is still behaving in a simple-jet-like matter, that with a similar linear growth for both the upper and lower region. When $S / D$ becomes much larger than $l_{M}$, the lower region, because of the unstable stratification, begins to widen with a faster rate than the upper region, whose widening is slowed down by the stable stratification (see paragraph 4.1).

To study the law of widening rate of the upper and lower region of these $\mathrm{NBJ}$, the upper region data have been fitted, on a least-square sense, with the linear law of eq. 8 , the lower boundary with both the eq. 8 and the $5 / 3$ power law of eq. 9 :

$$
\begin{aligned}
& \frac{R}{D}=c \frac{S}{D}+\frac{1}{e} \\
& \frac{R}{D}=\kappa\left(\frac{S}{D}\right)^{5 / 3}+\frac{1}{e}
\end{aligned}
$$

Obviously, the constant $1 / \mathrm{e}$ has been added because, as a consequence of the chosen definition for the widening, the NBJ width at the outlet has this value for all the $\mathrm{Fr}$ and $\theta$.

Looking at Figures from 7 to 10 , equation 8 seems to correctly fit the data relative to the upper region. Focusing on the lower region, both equations 8 and 9 are able to catch some of the data features, with some lacks in precision for the lowest and highest $S / D$. More in details, equation 8 strongly overestimates the initial widening of the lower region and underestimates the final growth. 


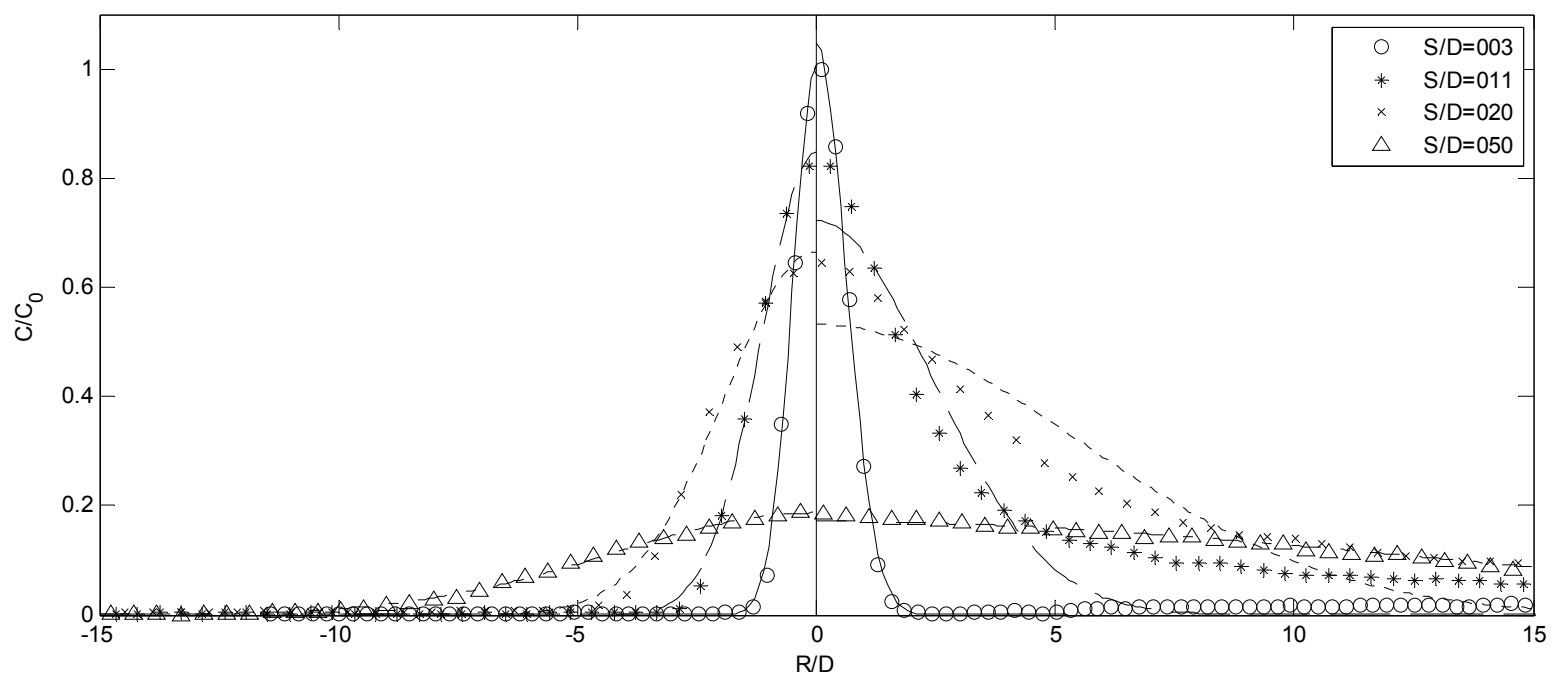

Fig. 6. Interpolation with best-fit (least mean squares) Gaussian curves of cross-sectional non-dimensional concentration profiles: on the left the upper region of the NBJ, on the right the lower region of the NBJ (same NBJ as in Figures 4 and 5).

Equation 9, instead, slightly underestimates the initial widening (and it better follows the trend of the data for low values of $S / D$ ) but it is able to better catch the trend for increasing $S / D$, particularly for the lowest Frs. As a consequence, we can state that equation 9 seems to better fit the points with the highest $S / D$, which could mean that, if the nearest region to the origin is not taken into account, the lower region tends to a faster than linear evolution or, in other words, doesn't behave like a simple jet of a plume (both linear).

It seems, thereby, that the upper boundary of the inclined NBJs tends to develop following a linear law, whilst the lower boundary tends to evolve in a more complex way, nor jet- neither plume-like, in particular when $S / D$ increases and becomes much larger than $l_{M}$. On Table 1, the coefficient of eq. 8 and 9 for the curves of Figures 7, 8, 9 and 10 have been reported; the subscript $U$ refers to the upper region, the subscript $L$ to the lower region. The values of the coefficient $c_{U}$ (representing the rate of growth of the upper part of the inclined NBJs) decrease with decreasing $F r$, suggesting that, as the NBJs become heavier, the stable stratification on the upper boundary prevents the Kelvin-Helmholtz billows to develop vertically and, consequently, the NBJs to widen more. On the opposite, the coefficients $\kappa$ increase with decreasing $F r$, suggesting that, as the NBJs become heavier, the unstable stratification on the lower region helps the descending plumes to detach and, as a consequence, the NBJs to widen more. Given a fixed $S / D$, as $F r$ decreases (i.e., $B$ increases), the lower region of an inclined NBJ tends to widen more whilst, viceversa, the upper boundary tends to widen less.

Finally, considering all the showed results, we can state that an incline NBJ cannot be considered as an axisymmetric or Gaussian phenomenon, as some features of simple jets, plumes and more complex ones coexist: the upper region tends to evolve in a simple-jetlike way, following a linear law widening rate; the lower boundary in a more complex manner, following a power law widening rate. The investigation on inclined NBJs can be considered, consequently, a complicated one, because we are not allowed to employ the well-known integral equations usually adopted to forecast the behaviour of axisymmetric jets or plumes.

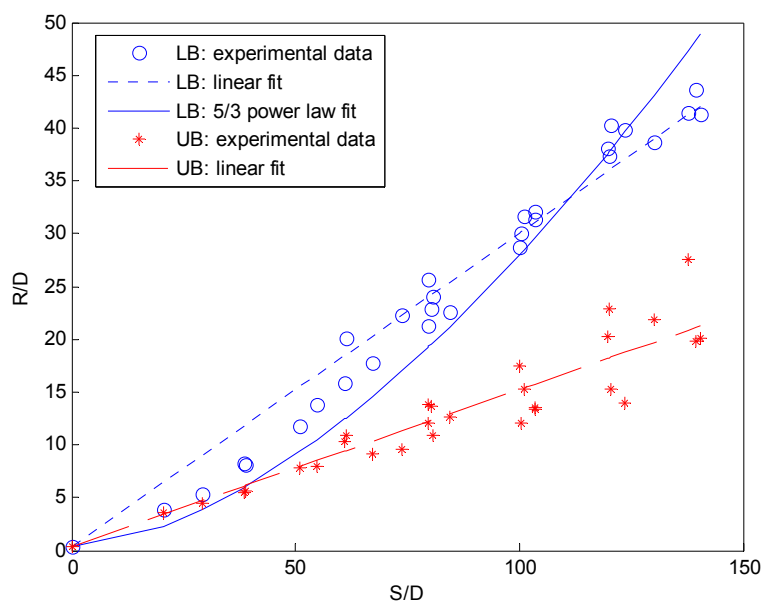

Fig. 7. Experimental data for the widening of the upper boundary (UB) and lower boundary (LB) of NBJs with $\mathrm{Fr}=$ 30.8 and $\theta=45^{\circ} \div 70^{\circ}$.

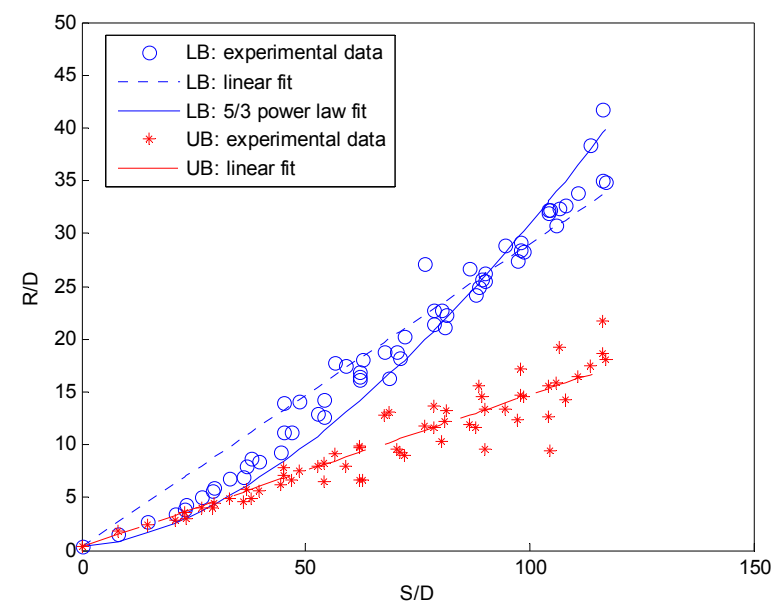

Fig. 8. Experimental data for the widening of the upper boundary (UB) and lower boundary (LB) of NBJs with $F r=$ 23.8 and $\theta=45^{\circ} \div 70^{\circ}$.

Corresponding author: ferraris@unica.it 


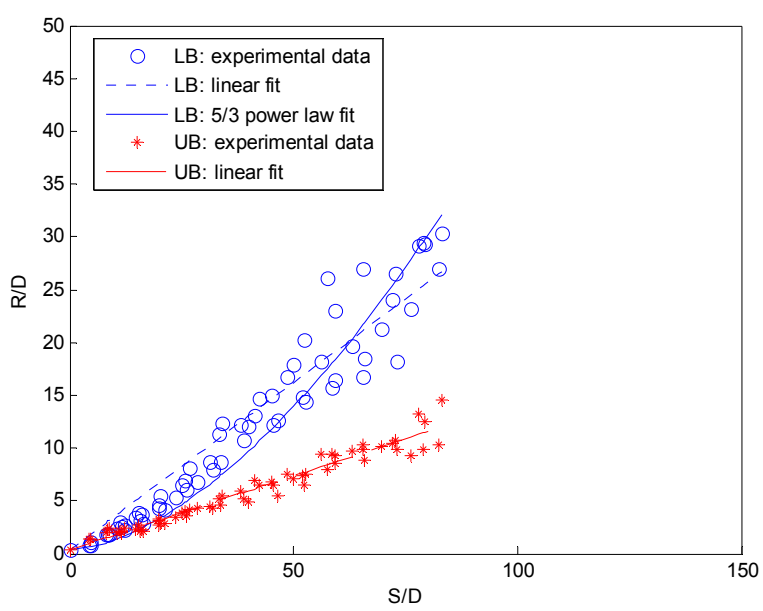

Fig. 9. Experimental data for the widening of the upper boundary (UB) and lower boundary (LB) of NBJs with $F r=$ 14.8 and $\theta=45^{\circ} \div 70^{\circ}$.

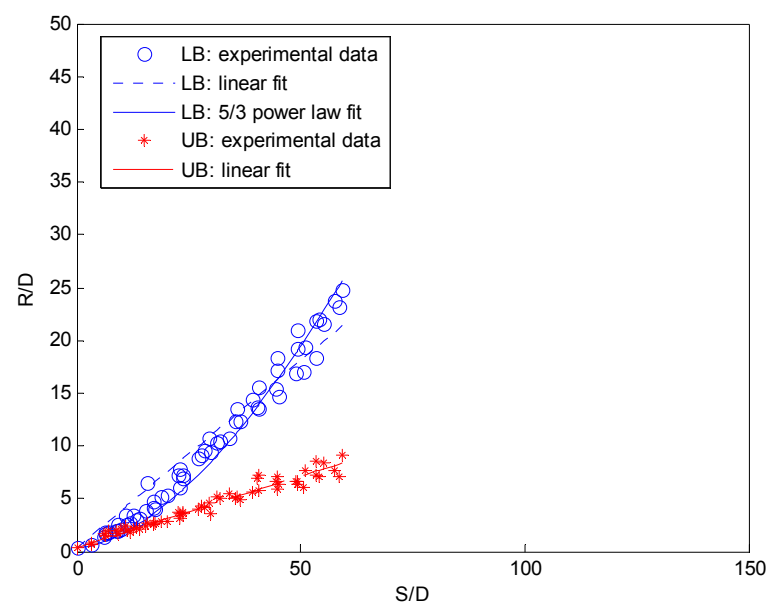

Fig. 10. Experimental data for the widening of the upper boundary (UB) and lower boundary (LB) of NBJs with $F r=$ 8.0 and $\theta=45^{\circ} \div 70^{\circ}$.

Table 1. coefficients of the eq. 6 and 7 for the data of Figure 5; $\mathrm{U}=$ upper boundary, $\mathrm{L}=$ lower boundary.

\begin{tabular}{|c|c|c|c|}
\hline $\boldsymbol{F r}$ & $\boldsymbol{c}_{\boldsymbol{L}}$ & $\boldsymbol{\kappa}$ & $\boldsymbol{c}_{\boldsymbol{U}}$ \\
\hline $\mathbf{3 0 . 8}$ & 0.297 & 0.013 & 0.149 \\
\hline $\mathbf{2 3 . 8}$ & 0.286 & 0.014 & 0.143 \\
\hline $\mathbf{1 4 . 8}$ & 0.317 & 0.020 & 0.140 \\
\hline $\mathbf{8 . 0}$ & 0.355 & 0.028 & 0.136 \\
\hline
\end{tabular}

\section{Conclusions}

In order to have a better insight into the effects of stable and unstable stratification on NBJs released with a certain angle to the horizontal $\theta$, an experimental investigation have been carried out in the Hydraulic Laboratory of the DICAAR, University of Cagliari. A non-intrusive Digital Image Analysis technique, namely LIF (Light Induced Fluorescence), was in-house developed and employed. The experimental parameters, in particular $\theta$ and the densimetric Froude number $F r$, were chosen in order to investigate the whole field of practical interest for the marine brine discharge.
The results show that the different conditions of stratifications, in particular a stable stratification on the upper region and an unstable stratification on the lower region, force the lower region of the NBJ to widens with a faster rate than the upper region, leading to a strongly asymmetric and non-Gaussian phenomenon. As a consequence, a great care should then be employed when applying the integral equations for axisymmetric jets or plume to inclined NBJs. Moreover, the experimental data have shown that the upper region has a linear widening rate, whilst the lower region tends to widen with a faster $5 / 3$ power law rate.

\section{Acknowledgement}

The Authors would like to acknowledge Dr. Luigi Antonio Besalduch, Dr. Michela Garau, Mr. Antonio Mascia, Dr. Lionel Rossi and Prof. John Christos Vassilicos.

\section{References}

1. Wang, S., Wang, Y., Zhuo, W., Niu, F., Yu, Y., Guo, Z., Shen, Y., Chen, W., Jiang, X., Experimental analysis of steam mixing and thermal stratification phenomena related to small steel containment studies, Ann. Nucl. En. 109, pp 103112 (2017)

2. Kaminski, E., Tait, S., Carazzo, G., Turbulent entrainment in jets with arbitrary buoyancy, J. Fluid Mech., 526, pp. 361-376 (2005)

3. Ferrari, S., Querzoli, G., Mixing and re-entrainment in a negatively buoyant jet, Journal of Hydraulic Research, 48 (5), pp. 632-640. (2010)

4. Cipollina, A., Bonfiglio, A., Micale, G. Brucato A., Dense jet modeling applied to the design of dense effluent diffusers, Desalination, 167, pp. 459-468 (2004)

5. Kikkert, G.A., Davidson, M.J., Nokes, R.I. Inclined negatively buoyant discharges. J.Hydr.Eng 133(5), pp. 545-554 (2007)

6. Besalduch, L.A., Badas, M.G., Ferrari, S., Querzoli, G., Experimental Studies for the characterization of the mixing processes in negative buoyant jets, EPJ Web of Conferences, 45, art. no. 01012 (2013)

7. Ferrari, S., Querzoli, G., Laboratory experiments on the interaction between inclined negatively buoyant jets and regular waves, EPJ Web of Conferences, 92, art. no. 02018 (2015)

8. Crowe, A.T., Davidson, M.J., Nokes, R.I., Velocity measurements in inclined negatively buoyant jets, Environ Fluid Mech, 16, pp. 503-520 (2016)

9. Nikiforakis, I.K., Stamou, A.I., Christodoulou, G.C., Integrated modeling of single port brine discharges into unstratified stagnant ambient, Environ Fluid Mech, 17, pp. 247-275 (2017)

10. Campbell, R.L., Jones, A.T., Appropriate disposal of effluent from coastal desalination facilities, Desalination, 182, pp. 365-372 (2005) 
11. Latorre, M., Environmental impact of brine disposal on Posidonia seagrasses, Desalination, 182, pp. 517524 (2005)

12. Sànchez-Lizaso, J.L., Romero, J., Ruiz, J., Gacia, E., Buceta, J.L., Invers, O., Fernàndez Torquemada, Y., Mas, J., Ruiz-Mateo, A., Manzanera, M., Salinity tolerance of the Mediterranean seagrass Posidonia Oceanica: recommendations to minimize the impact of brine discharges from desalination plants, Desalination, 221, pp. 602-607 (2008)

13. Roberts, P.J.W., Toms, G., Ocean outfall system for dense and buoyant effluents, J.Environ.Eng., 114(5), pp. 1175-1191 (1988)

14. Jirka, G.H., Improved Discharge Configurations for Brine Effluents from Desalination Plants, J.Hydr.Eng., 134, pp. 116-120 (2008)

15. List, E.J., Turbulent jets and plumes, in: Fisher, H.B., List, E.J., Koh, R.C.Y., Imberger, J., Brooks, N.H., (eds), Mixing in inland and coastal water. New York, USA, Academic Press, pp. 315-389 (1979)

16. Wright, S.J., Wong, D.R., Zimmerman, K.E., Wallace, R.B., Outfall diffuser behaviour in stratified ambient fluid, J.Hyd.Div. ASCE, 108(4), pp. 483-501 (1982)

17. Avanzini, C., An outline of common practical problems and solutions in the diffusers design, in: Avanzini, C. (ed.), Proceedings of MWWD \& IEMES 2006, Antalya (Turkey), 6-10 November 2006, ISBN: 9944-5566-0-2 (2006)

18. Ferrari, S., Image analysis techniques for the study of turbulent flows, EPJ Web of Conferences, 143, art. no. 01001 (2017)

19. Ferrari, S., Badas, M.G., Querzoli, G., A nonintrusive and continuous-in-space technique to investigate the wave transformation and breaking over a breakwater, EPJ Web of Conferences, 114, art. no. 02022 (2016)

20. Besalduch, L.A., Badas, M.G., Ferrari, S., Querzoli, G., On the near field behavior of inclined negatively buoyant jets, EPJ Web of Conferences, 67, art. no. 02007 (2014)

21. Ferrari, S., Badas, M.G., Garau, M., Seoni, A. Querzoli, G., The air quality in narrow twodimensional urban canyons with pitched and flat roof buildings, Int. J.Environment and Pollution, in press (2017)

22. Ferrari, S., Rossi, L., Vassilicos, J.C., Acceleration measurements in turbulent-like flows, Advances in Turbulence XI - Proceedings of the 11th EUROMECH European Turbulence Conference, pp. 485-487 (2007)

23. Ferrari, S., Rossi, L., Particle tracking velocimetry and accelerometry (PTVA) measurements applied to quasi-two-dimensional multi-scale flows, Experiments in Fluids, 44 (6), pp. 873-886 (2008)

24. Ferrari, S., Kewcharoenwong, P., Rossi, L., Vassilicos, J.C., Multi-scale flow control for efficient mixing: Laboratory generation of unsteady multi-scale flows controlled by multi-scale electromagnetic forces, Solid Mechanics and its Applications, 7, pp. 267-272 (2008)
25. Lardeau, S., Ferrari, S., Rossi, L., Threedimensional direct numerical simulation of electromagnetically driven multiscale shallow layer flows: Numerical modeling and physical properties, Physics of Fluids, 20 (12), art. no. 127101 (2008)

26. Rossi, L., Bocquet, S., Ferrari, S., Garcia de la Cruz, J.M., Lardeau, S., Control of flow geometry using electromagnetic body forcing, International Journal of Heat and Fluid Flow, 30 (3), pp. 505-513 (2009)

27. Badas, M.G., Espa, S., Fortini, S., Querzoli, G., 3D Finite Time Lyapunov Exponents in a left ventricle laboratory model, EPJ Web of Conferences, 92, art. no. 02004 (2015)

28. Espa, S., Badas, M.G., Fortini, S., Querzoli, G., Cenedese, A., A Lagrangian investigation of the flow inside the left ventricle, European Journal of Mechanics, B/Fluids, 35, pp. 9-19, (2012)

29. Badas, M.G., Ferrari, S., Garau, M., Querzoli, G., On the effect of gable roof on natural ventilation in two-dimensional urban canyons, J Wind Eng Ind Aerodyn 162, pp. 24-34 (2017) 